\title{
To compare the Safety \& Efficacy of Platelet Rich Plasma Therapy plus Narrowband Ultravoilet B (NBUVB) therapy versus Narrowband Ultravoilet B Therapy alone in the treatment of Vitiligo - A Double Blind, Randomised Controlled Study
}

Authors

\author{
Mohan S Kale, Dhanraj D Chavan, Varsha P Jamale, Balkrishna P Nikam*, \\ Asma Hussain, Ria Rai, Shruti D Chavan \\ Dept of Dermatology, Krishna Institute of Medical Sciences, Karad, MH, India \\ *Corresponding Author \\ Balkrishna P Nikam \\ Dept of Dermatology, Krishna Institute of Medical Sciences, Karad MH, India \\ Mob - 8692999088, Email: drdhanrajchavan@gmail.com
}

\begin{abstract}
Phototherapy with narrowband ultraviolet $B(N B U V B)$ therapy is the gold standard treatment for the treatment of vitiligo currently. The long duration of treatment is one of the major limiting factors of the therapy. Platelet Rich Plasma (PRP) is one of the promising new treatments in the arena of dermatology and has been previously tried by some authors with varying amounts of success in the treatment of vitiligo. In this study we have attempted to analyse whether the addition of PRP therapy to NBUVB therapy in the treatment of vitiligo provides any advantage in terms of the repigmentation speed. We concluded at the end of the study that addition of PRP to NBUVB therapy does not provide any advantage for faster outcomes of treatment.

Keywords: PRP, NBUVB, Vitiligo.
\end{abstract}

\section{Introduction}

Pearson, just after the end of the nineteenth Century described under the name leukoderma, a disease similar to vitiligo. In the late twentieth century, the elusive nature of vitiligo has led to prudent definitions excluding disorders of established aetiology. ${ }^{[1]}$

Vitiligo is classified according to Picardo and Taieb into four types: Non-segmental vitiligo (NSV), segmental vitiligo (SV), mixed NSV and SV, and unclassifiable types e.g., focal, multifocal asymmetrical non-segmental and mucosal at one site. ${ }^{[2]}$
The aim of treatment in vitiligo is to restore the normal appearance, morphology, and function of skin. Narrowband UVB has become the first-line treatment for adults. Platelet-rich plasma (PRP) (syn. autologous platelet gel, plasma-rich growth factors and platelet-concentrated plasma) means "abundant platelets that are concentrated into a small volume of plasma."

Aim
To determine the effect of Platelet Rich Plasma
(PRP) injection on the outcome of narrow band-
ultraviolet B (NB-UVB) phototherapy for the
patients with non segmental stable vitiligo. 


\section{Materials and Methods}

Study Type: The present study was a prospective, double blind, randomized controlled study.

Study Settings: The study was conducted in the outpatient department of Dermatology in a tertiary care hospital in Karad, India.

Study Duration: The total study duration was 24 months, from November 2016 to October 2018. Each patient was treated for 4 months, which included bimonthly sessions of local injection of PRP or normal saline and biweekly sessions of NV-UVB exposure and the results analysed.

Sample Size: The sample size of 32 patients with 64 vitiligo patches (32 patches each of Control and Experimental group) was calculated with the help of Open Epi, Version 3, open source calculator.

Inclusion Criteria: Cases of vitiligo with overall symmetrical stable lesions, with

- absence of new areas of depigmentation for 12 months

- no enlargement of the preexisting lesions for 12 months

- absence of Koebner phenomena for 12 months

\section{Exclusion Criteria}

- Patients who developed new lesions in the past one year.

$\circ$ Patients with segmental or extensive generalized Vitiligo

- Patients who are known to have a good or rapid response to conventional modalities

- Pregnant or lactating females and young patients below 18 years

\section{Methods}

A total of 32 patients were enrolled in the present study from the Outpatient Department of Dermatology of a tertiary care hospital in Karad, Maharashtra, India, during the period from November 2016 to June 2018 after obtaining the approval of the research ethics committee. A detailed informed written consent was taken from the patients.

For each patient, a lesion on one side of the body was treated with NB-UVB along with intradermal injection of PRP (case side) while the other side was treated with NB-UVB therapy along with intradermal injections of normal saline (control side). The NB-UVB therapy was given biweekly and intradermal injections of PRP and NS was given bimonthly. Each lesion of approximately palm size of the patient, along with a similar lesion on the opposite side was considered as two samples. Patients were photographed, at the first visit, at the end of $2^{\text {nd }}$ month and at the end of $4^{\text {th }}$ month.

\section{Randomization of Cases \& Controls}

The allocation of sides of the body to be injected with PRP and NS control were decided by random picking of pre labeled cards with sides mentioned, stored in a box.

\section{$>$ NB-UVB therapy}

All patients received biweekly NB-UVB sessions for 4 months, with the option of continuing the therapy to completion. The NB fluorescent tubes (Philips TL 100, Hamburg, Germany) with a spectrum of 310-315 $\mathrm{nm}$ and a maximum wave length of $311 \mathrm{~nm}$ were the NB-UVB source. The initial dose of UVB was $330 \mathrm{~mJ} / \mathrm{cm} 2$, and increased by $20 \%$ every session till the minimal erythema dose was achieved.

\section{> Platelet-rich plasma separation method}

To create PRP, $20 \mathrm{cc}$ of venous blood was collected under complete aseptic conditions. The whole blood sample was collected in sterile test tubes and ACD (Acid Citrate Dextrose) was added to it as an anticoagulant. Then the test tubes were subjected to two centrifugation steps. The initial centrifugation was done ("soft" spin) at $1500 \mathrm{rpm}$ for $15 \mathrm{~min}$ to separate the plasma and platelets from the red and white cells. The resulting plasma supernatant, which contained the suspended platelets will be harvested to a second centrifugation step ("hard" spin) at $2000 \mathrm{rpm}$ for 9 min, leading to separation of the plasma into 2 portions:- platelet-poor plasma (PPP) and PRP. Typically, the lower $2 \mathrm{cc}$ of the plasma (10\% of the initial volume of autologous blood), yielded as 
PRP concentrate after centrifugation. It was injected intradermally using insulin syringe in the lesion, $0.1 \mathrm{cc}$ of PRP was injected per point with a space of $2 \mathrm{~cm}$ between different points of injections. This procedure was repeated every 2 weeks for every patient for upto 4 months (8 sessions).

\section{> Injection of Normal Saline on Control side}

In order to blind the patient, and to act as a control for the microneedling effect of PRP, the lesions of control side, $0.1 \mathrm{cc}$ of NS was injected intradermally per point with a space of $2 \mathrm{~cm}$ between different points of injections.

The patients were informed to report any complications as; erythema, pain, ulceration, burning sensation, ecchymosis, infection, post inflammatory hyperpigmentation, or any allergic manifestations. The emergence of adverse effects such as ulceration was a guideline to stop the NBUVB therapy, or dose adjustments.

\section{$>$ Follow-up Assessment}

The patients were examined in the first visit and were reviewed bimonthly for the progress of therapy and the presence of any side effects. Evaluation of pigmentation was done by two independent dermatologists. Photographs were taken at baseline, middle ( $5^{\text {th }}$ visit) and end of treatment period $\left(9^{\text {th }}\right.$ visit)

The repigmentation response was rated qualitatively as follows by a physician global assessment (PGA) of the Repigmentation response:

Table 1: Physician global assessment scale

\begin{tabular}{|l|c|}
\hline Score & Scale \\
\hline 0 & Poor \\
\hline 1 & Mild \\
\hline 2 & Moderate \\
\hline 3 & Good \\
\hline 4 & Excellent \\
\hline
\end{tabular}

\section{Statistical Analysis}

The data was collected using a pre-validated, standard case record proforma. The data was entered and tabulated with the help of Microsoft Excel software. The data was analyzed to derive means, standard deviations, and levels of significance using SPSS Version-21 software. The difference between two means of change in depigmented area was statistically analyzed using the Student's $\mathrm{T}$ test (Parametric tests). The qualitative improvement data was analysed using the chi square test (Non-parametric tests)

\section{Results \\ Demographic Characteristics \\ - Gender Distribution}

We assessed demographic profiles of the study participants (Cases of vitiligo). Out of total 32 cases, Number of female cases - 19 (59.37\%) outnumbered male cases - 13 (40.62\%)

\section{- Age Distribution}

We included cases of generalized vitiligo, above the age of 18 years. Further we studied their agewise distribution, andfound that the majority of the study participants belonged to the age group of $36-45$ years [ 9 cases $(28.12 \%)$ ] followed by $26-35$ years [8 cases $(25 \%)]$ and then $46-55$ years [5 cases $(15.62 \%)]$. Cases were less in number above the age of 65 years [2 cases $(6.25 \%)$ ]

\section{Physician Global Assessment Scale}

Similarly, the physician's rating for the repigmentation response was rated and compared on Physician global assessment scale:

Responses were rated as Excellent, Good, Moderate, Mild and Poor among 6.25\%, 12.5\%, $34.37 \%, 37.5 \%$ and $9.37 \%$ cases respectively in PRP group, whereas it was $3.12 \%, 15.62,37.5 \%$, $31.25 \%$ and $12.5 \%$ respectively in control group. The difference of response rates between both groups was not found statistically significant ( $\mathrm{P}$ value $>0.05$ ). (Figure 18) 
Figure 18: Distribution of study population according to the response after treatment according to the Physician global assessment

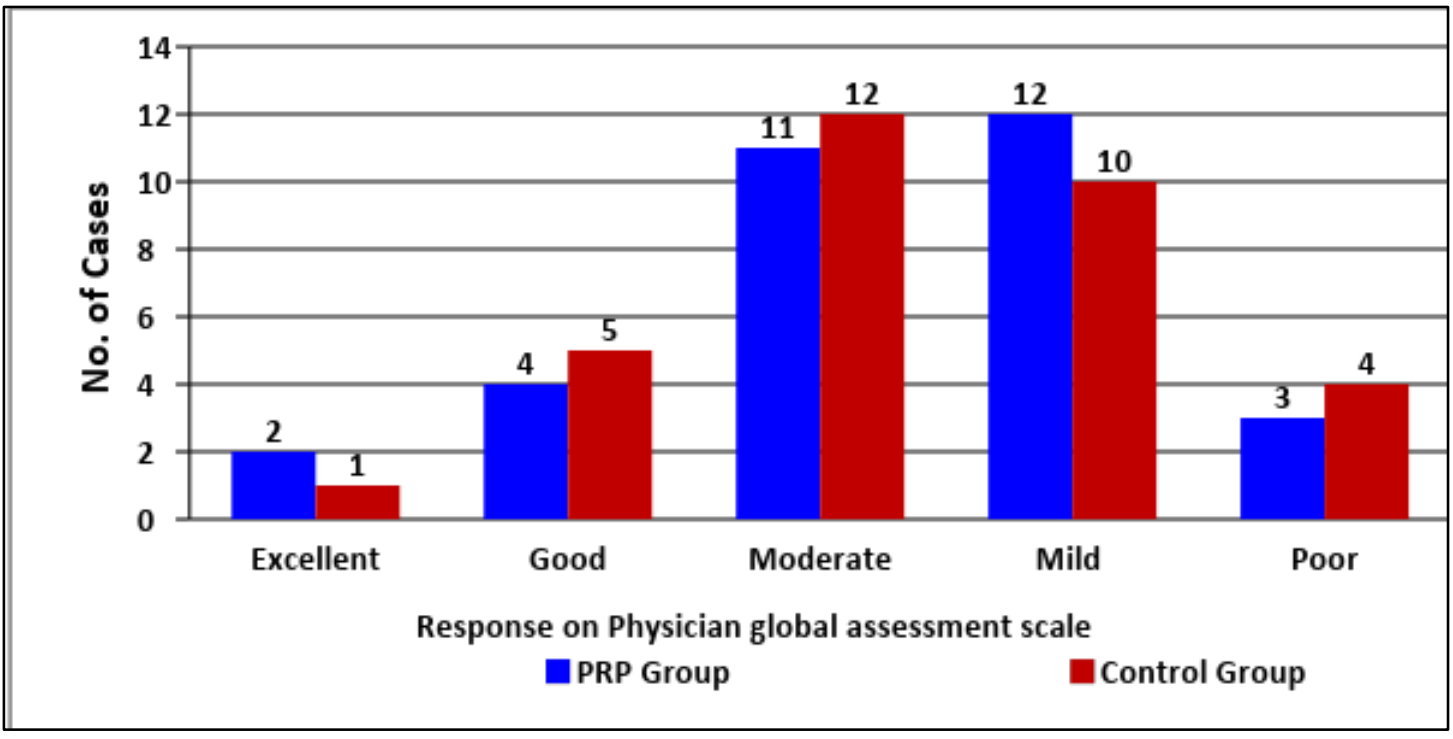

\section{Side Effects Associated with the Treatment}

In the present study, we studied side effects that were experienced during the treatment. In the PRP group, we injected PRP in the lesion. In order to eliminate the bias, we injected normal saline in the control group.

Pain during injection of normal saline was observed to be the most frequently experienced side effect during the treatment. 15 (46.87\%) cases in PRP group complained of pain while injecting the PRP while the number of participants complaining of pain in control group was slightly more i.e. 17 (53.12\%)

Ecchymosis was observed among 3 cases $(9.37 \%)$ treated with PRP and 2 cases $(6.25 \%)$ treated with NB-UVB alone (control group).

\section{Photographs of Lesions Treated with PRP + NBUVB}

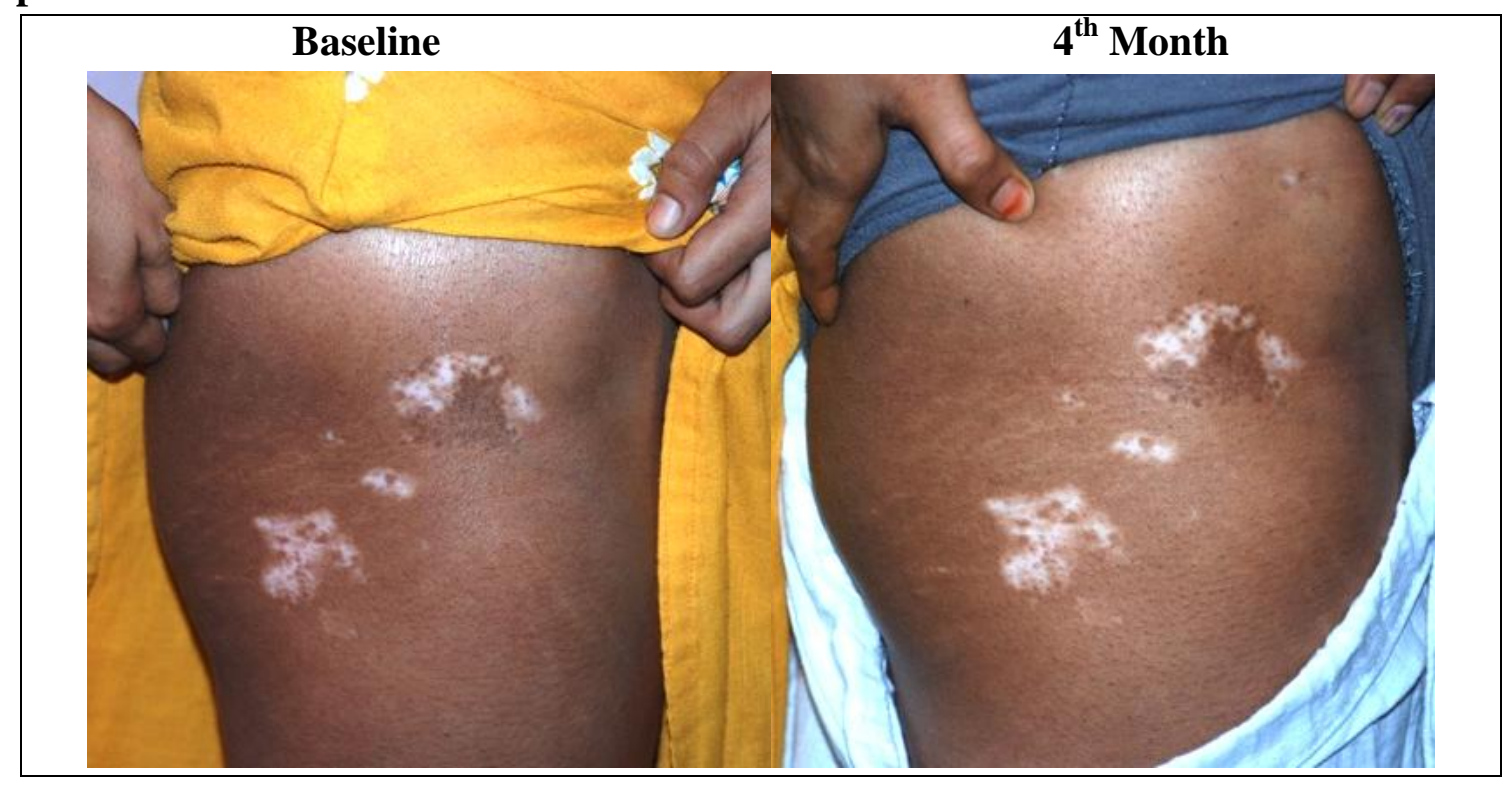




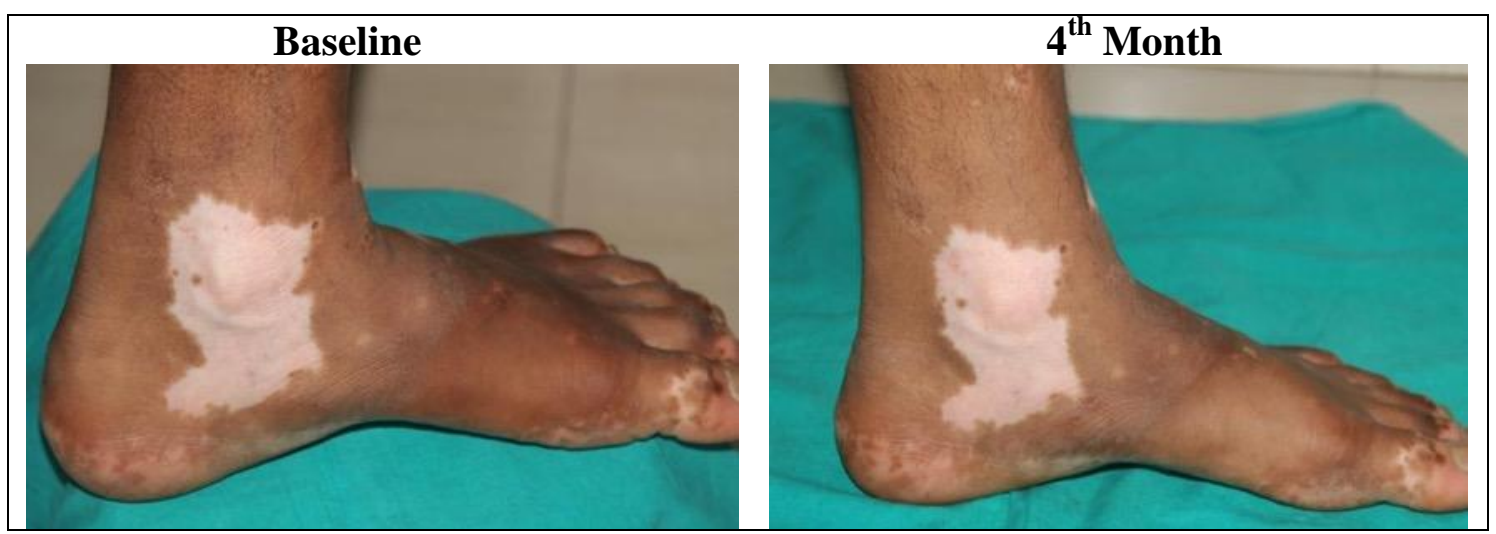

Photographs of Lesions Treated with NS + NBUVB

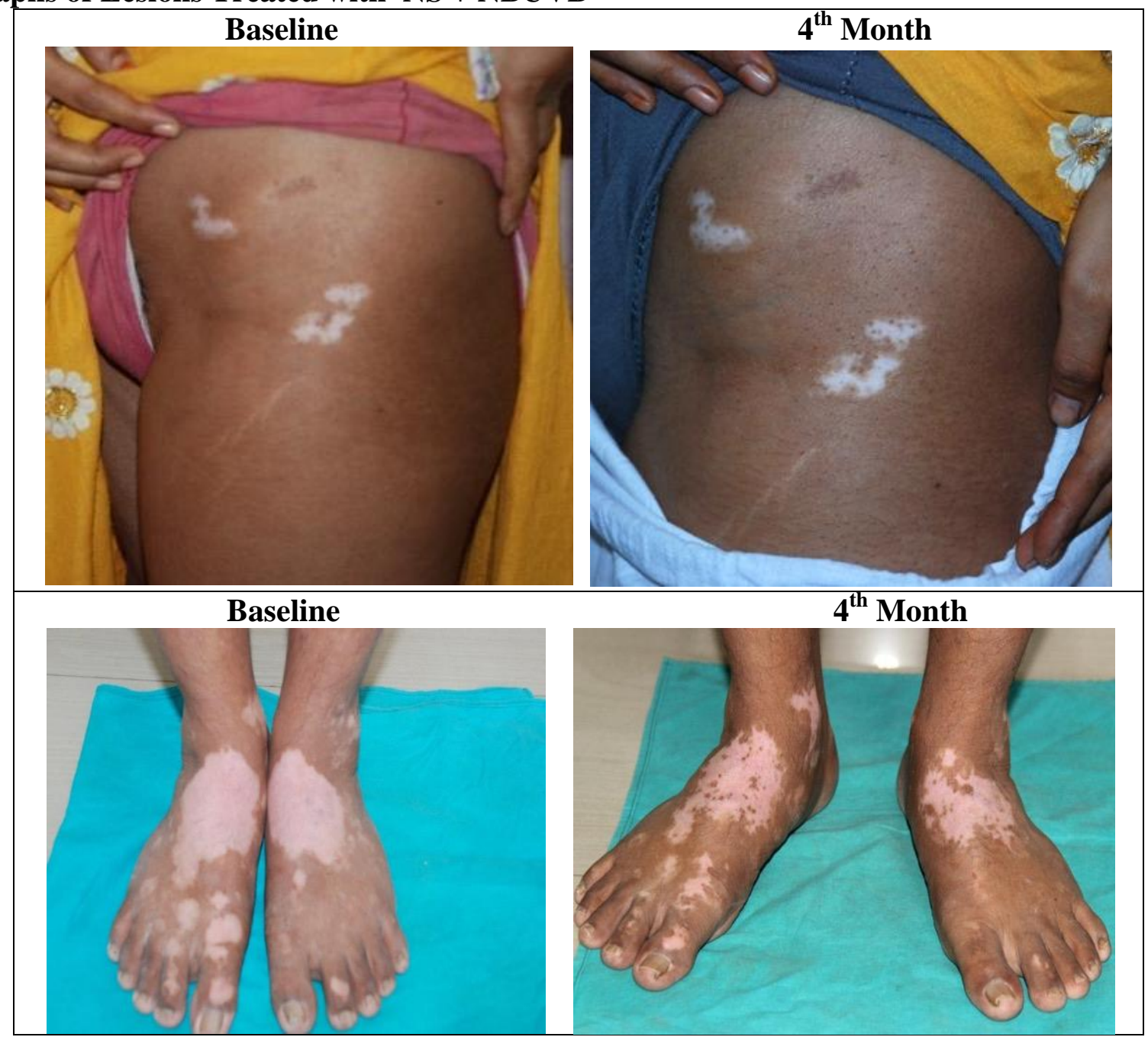

\section{Discussion}

The aim of treatment in vitiligo cases is to restore the normal appearance, morphology, and function of skin. Several treatment modalities are currently available; each having certain indications and limitations.

\section{Demographic features}

We assessed demographical characteristics of study participants. Majority of the cases were females $(59.37 \%)$ as compared to male cases. Imran Majid et $\mathrm{al}^{[4]}$, in their study, also found female sex as preponderant (14 males in comparison with 26 females). Kishan Kumar et $\mathrm{al}^{[3]}$ in their study among 150 cases of vitiligo, observed 69 males, 81 females, aged 3-70 years,

We studied age distribution of the study participants, we found that the majority of the study participants belonged to the age group of 
36-45 years followed by 26-35 years and then 4655 years. Rashmi Mahajan et al ${ }^{[5]}$ in their study observed 18 males (45\%) and 22 females (55\%). Their ages were from 12 to 40 years.

\section{Interventions}

\section{Outcome indicators: Re-pigmentation response Physician Global Assessment Scale}

In the present study, the repigmentation response was again compared with the help of Physician global assessment scale. Responses ranged from Excellent, Good, Moderate, Mild and Poor among $6.25 \%, 12.5 \%, 34.37 \%, 37.5 \%$ and $9.37 \%$ cases respectively in PRP group. Whereas it was $3.12 \%$, $15.62,37.5 \%, 31.25 \%$ and $12.5 \%$ respectively in control group. The difference between findings of both groups was not found statistically significant ( $\mathrm{P}$ value $>0.05)$.

Rashmi Mahajan et al ${ }^{[5]}$ in their study observed that out of 40 patients, 15 patients showed good response, 12 patients showed Average response and 13 patients showed no-response to treatment. The average increase in vitiligo area severity index (VASI) ranged from 100 to 10. Visible signs of improvement were after the 3rd injection. (6 weeks). Facial lesions responded very well with complete clearance of smaller lesions.

\section{Role of PRP Vs NB-UVB}

This present study compares outcomes in the form of re-pigmentation response, after treatment with NB-UVB on one side and combination of PRP and NB-UVB on other side. We rated outcomes qualitatively, using physicians global assessment scale.

Though this study observes better re-pigmentation among the combination of NB-UVB and PRP side (study side), the differences were not found statistically significant.

Ibrahim et al., conducted a study in 2015 in Egypt, they evaluated the effect of platelet-rich plasma on the outcome of short-term narrowband-ultraviolet B phototherapy in the treatment of vitiligo was evaluated with regard to body site and it was found that vitiligo of the face region, trunk areas and extremities showed the best results. However in our study facial lesions showed a better response than truncal lesions and extremities showed a poor response. In the same study conducted by Ibrahim et al, 55\% of patients in the PRP group achieved excellent repigmentation and $20 \%$ achieved good repigmentation in a 4-month duration compared to NB-UVB side. They reported that the combination group (PRP plus NB-UVB) had a statistically significant improvement in repigmentation, compared with the NB-UVB group, with pigmentation detected in $43.3 \%$ of patients in the combined side versus $10 \%$ in the NB-UVB side after the third session. According to them intradermal PRP injection in combination with NB-UVB could be considered as a simple, safe, and not expensive technique for treatment of vitiligo. It also reduced the duration of NB-UVB therapy and is expected to increase patient compliance. In our study $37.5 \%$ patients showed good response and $30 \%$ showed average response. The reported side effects in their study were minor in severity and all patients in the study tolerated the procedure well. Pain occurred in $50 \%$ of the patients which was mild and tolerable. Ecchymosis at the site of injection occurred in $15 \%$.

In the year 2014, Ahmad Nassar et al ${ }^{[7]}$ conducted a Prospective Open Pilot Study to evaluate the efficacy of PRP in the treatment of Vitiligo among 12 cases of vitiligo, who received intradermal PRP once in 2 weeks for 12 sessions or till improvement occurred. All patients in their study showed repigmentation (100\%) with variable VETF scores. The best response was after a mean \pm SD of $9.38 \pm 2.48$ (Median=10) sessions. According to them Autologous PRP could be considered as an effective therapy with minimal side effects and cost effective measure.

Rania Abdelghani et $\mathrm{al}^{[8]}$ conducted a comparative study among 80 adult patients with localized nonsegmental vitiligo. They categorized the cases randomly into 4 lines to receive lines of treatment; fractional CO laser, PRP, combined fractional $\mathrm{CO}$ laser and PRP, and combined fractional $\mathrm{CO}$ laser and NBUVB. They decided 
the treatment period as 2 months. Patients were clinically evaluated 3 months after the last treatment. Outcome was evaluated by 5-point scale for repigmentation, 10-point visual analog scale for patient's satisfaction, and side effects. They reported that the Laser and PRP group achieved the best results regarding repigmentation and patient's satisfaction. $60 \%$ patients developed repigmentation $>50 \%$ and $40 \%$ of patients developed repigmentation $>75 \%$. In laser and NBUVB group, 5\% developed repigmentation $>75 \%$ and $25 \%$ developed repigmentation $>50 \%$. Only $10 \%$ of patients developed repigmentation $>75 \%$ in laser group and only $20 \%$ of patients developed repigmentation $>75 \%$ in PRP group. At the end they deduced that Combination of fractional $\mathrm{CO} 2$ laser with PRP injection is a promising treatment for vitiligo, followed by combination of fractional $\mathrm{CO} 2$ laser with NB-UVB phototherapy. Both fractional $\mathrm{CO} 2$ laser and PRP injection gave poor results if they received alone.

\section{Summary}

$>$ Through our study, we attempted to analyse whether the addition of PRP therapy has any advantage over the conventional NBUVB therapy in fastening the repigmentation rates in cases of Vitiligo.

$>$ We did this by comparing the rates over two lesions on the same patient, one injected with PRP and the other with control injections of Normal saline. A total of 32 patients \& 64 lesions were included in the study.

$>$ We assessed the repigmentation with the help of qualitative repigmentation parameters

$>$ We found that addition of PRP has no statistically significant advantage in treating patients of stable vitiligo.

\section{Limitations}

The limitations of our study include:

- The shorter treatment duration period,
- The paucity of patient follow up post treatment completion

\section{Conclusions}

From the present study. we conclude that though PRP may have some role to play in the faster repigmentation seen in some patients, the statistical tests do not show a significant advantage over the control group.

\section{Bibliography}

1. P. Manga, N. Elbuluk, and S. J. Orlow, "Recent advances in understanding vitiligo.," F1000Research, vol. 5, 2016.

2. J. Kanwar and M. S. Kumaran, "Childhood vitiligo: treatment paradigms.," Indian J. Dermatol., vol. 57, no. 6, pp. 466-74, Nov. 2012.

3. Y. H. Kishan Kumar, G. R. R. Rao, K. V. T. Gopal, G. Shanti, and K. V. Rao, "Evaluation of narrow-band UVB phototherapy in 150 patients with vitiligo.," Indian J. Dermatol. Venereol. Leprol., vol. 75, no. 2, pp. 162-6.

4. Majid, "Efficacy of targeted narrowband ultraviolet B therapy in vitiligo.," Indian J. Dermatol., vol. 59, no. 5, pp. 485-9, Sep. 2014.

5. R. Mahajan, K. Ninama, H. Shah, and F. Bilimoria, "Effect of intralesional platelet rich plasma in chronic localized vitiligo," Int. J. Res. Dermatology, vol. 4, no. 4, p. 550, Oct. 2018.

6. Z. A. Ibrahim, A. A. El-Ashmawy, R. A. El-Tatawy, and F. A. Sallam, "The effect of platelet-rich plasma on the outcome of short-term narrowband-ultraviolet B phototherapy in the treatment of vitiligo: a pilot study," J. Cosmet. Dermatol., vol. 15, no. 2, pp. 108-116, Jun. 2016.

7. Ahmed Nassar, "Efficacy of platelet rich plasma in the treatment of vitiligo | Request PDF," in Efficacy of platelet rich plasma in the treatment of vitiligo, 2014. 
8. R. Abdelghani, N. A. Ahmed, and H. M. Darwish, "Combined treatment with fractional carbon dioxide laser, autologous platelet-rich plasma, and narrow band ultraviolet B for vitiligo in different body sites: A prospective, randomized comparative trial," J. Cosmet. Dermatol., vol. 17, no. 3, pp. 365-372, Jun. 2018. 